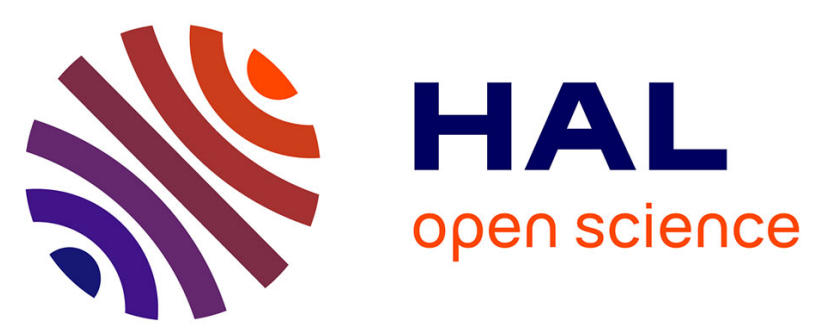

\title{
Nonlinear Modeling and Robust Controller-Observer for a Magnetic Microrobot in a Fluidic Environment using MRI gradients
}

\author{
Laurent Arcèse, Matthieu Fruchard, Antoine Ferreira
}

\section{- To cite this version:}

Laurent Arcèse, Matthieu Fruchard, Antoine Ferreira. Nonlinear Modeling and Robust ControllerObserver for a Magnetic Microrobot in a Fluidic Environment using MRI gradients. IEEE International Conference on Intelligent Robots and Systems, Oct 2009, Saint Louis, United States. pp 534-539. hal-00655982

\section{HAL Id: hal-00655982 https://hal.science/hal-00655982}

Submitted on 3 Jan 2012

HAL is a multi-disciplinary open access archive for the deposit and dissemination of scientific research documents, whether they are published or not. The documents may come from teaching and research institutions in France or abroad, or from public or private research centers.
L'archive ouverte pluridisciplinaire HAL, est destinée au dépôt et à la diffusion de documents scientifiques de niveau recherche, publiés ou non, émanant des établissements d'enseignement et de recherche français ou étrangers, des laboratoires publics ou privés. 


\title{
Nonlinear modeling and robust controller-observer for a magnetic microrobot in a fluidic environment using MRI gradients
}

\author{
Laurent Arcese, Matthieu Fruchard, Antoine Ferreira
}

\begin{abstract}
This paper reports the use of a MRI device to pull a magnetic microrobot inside a vessel and control its trajectory. The bead subjected to magnetic and hydrodynamic forces is first modeled as a nonlinear control system. Then, a backstepping approach is discussed in order to synthesize a feedback law ensuring the stability along the controlled trajectory. We show that this control law, combined with a high gain observer, provides good tracking performances and robustness to measurement noise as well as to some matched uncertainties.
\end{abstract}

\section{INTRODUCTION}

Microrobots designed to perform targeted therapy by navigating in the cardiovascular system are a prolific research area for minimally invasive surgery purposes [1]. Indeed, these untethered robots have the distinctive feature of being less invasive than catethered methods, and thus may reach remote parts of the human body that remain inaccessible to present tools without operating. On such a scale, it proves difficult to embed actuators sufficiently powerful to propel the robot, especially when swimming against the tide. Therefore, the great majority of swimming approaches considers magnetic fields to wirelessly transmit power to the robot [2], [3].

Untethered devices have been mainly developed according to three different designs: magnetic beads pulling, biomimetic flagellated robots and magnetotactic bacteria. The former propelling scheme relies on generating thrust on the microrobot using the magnetic force related to the gradients of the external field. This concept has first been studied using magnets [4] or superconducting magnets [5]. The last innovation in this domain has been provided by Martel [6], [7], where the basic idea is the use of the magnetic gradients coils of a clinical MRI to pull the robot. Since medical applications are the underlying theme of these works, using a clinical MRI device to actuate the robot combines several advantages. MRI devices are widely implanted and provide both a permanent magnetic field and magnetic gradient that can be used for propelling purposes. Besides, the MRI imager can provide fine observation of the scene for navigation and observation, using multiplexing for controlling and imaging [8]. In-vivo experiments have also been carried out [9]. The second class of robots, using respectively oscillating

L. Arcese and M. Fruchard are with the Institute PRISME EA 4229, IUT de Bourges, 63 Bd de Lattre de Tassigny, 18020, Bourges cedex, France laurent.arcesedbourges.univ-orleans.fr matthieu. fruchardebourges.univ-orleans. fr

A. Ferreira is with the Institute PRISME EA 4229 , ENSI de Bourges, 10 Bd Lahitolle, 18000, Bourges, France antoine.ferreiradensi-bourges.fr and rotating magnetic fields, imitates either the motion of a beating flagella [10] provided by bending and pivoting a magnetic particles made elastic rod, or the corkscrew effect of an helical coil attached to a magnetic core [11] to swim in Stokes flow. These techniques are well suited for nanorobots since they move at low Reynolds number, and are proved more efficient than bead pulling for robots whose radius is down to a few micrometers [12]. However, they will prove difficult to implement on classical MRI devices, since their propulsion requires a time-varying external field whereas actual MR imaging requires a uniform DC field. In [13], the flagella of magnetotactic bacteria have been exploited to provide propulsion, while steering has been achieved by inducing a torque on a chain of nanoparticles (magnetosomes) synthesized in the cell of each bacterium. Despite promising, this recent design faces some unsolved problems leading to reduced efficiency as reported in [13].

Efforts have mostly focused on fine modeling, feasibility and sizing of these various designs, but few works deal with advanced control and observation of these nonlinear systems. To our knowledge, the more advanced feedback controller scheme designed in the scope of microrobotics facing nonlinear drag is a PID approach developed in [8], [14]. Besides, there is no consideration about observation issues. Authors report in [8] instabilities and important oscillations around the equilibrium, especially when the blood stream is modeled as a pulsatile flow. They also indicate a lack of robustness to noise and unmodeled dynamics. However, it is clear that nonlinear control techniques can improve the tracking, both at the control and at the observation point of view.

The paper is organized as follows. Section II is devoted to the 2D-state space nonlinear model for a microrobot subjected to hydrodynamic and magnetic forces. In Section III, we synthesize a backstepping controller based on the previous model. Then Section IV is devoted to the high gain observer used to reconstruct the state, and especially the unmeasured robot's velocity that is needed to implement the backstepping control law. In Section V, simulation experiments are presented for both swimming with and against the flow and for bifurcating in an Y-shaped vessel. These simulations point out the robustness of the observer-controller pair to noise measurements and to blood's velocity uncertainties. Finally, conclusion and future works are detailed in the last section.

\section{MODELING}

Modeling at micro and nanoscale has been widely investigated. The purpose of this section is to get a basic state 
space model at microscale. Since the control is designed to make the robot levitate, it is not necessary to take friction forces into account in a first approximation. Moreover, we assume the microrobot is big enough to be unaffected by specific nanoforces.

\section{A. Forces Balance}

The microrobot immersed in a blood vessel is modeled by a ferromagnetic sphere with a high saturation magnetization. In this paper, the core is supposed to be made of permendur. Assuming the robot is spherical, with a some hundreds micrometers radius $r$, we now detail the forces acting on it, see Figure 1.

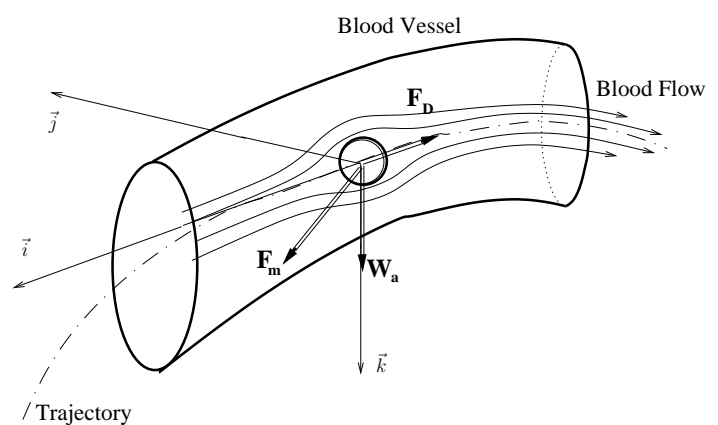

Fig. 1. Forces exerted on the core

1) Hydrodynamics: The Navier-Stokes equation allows to model hydrodynamics phenomena:

$$
\rho \frac{\partial \vec{v}}{\partial t}+\rho(\vec{v} \cdot \nabla) \cdot \vec{v}=\vec{f}-\nabla p+\nabla \cdot \sigma
$$

where $\rho$ denotes the density of the fluid, $\vec{v}$ the fluid velocity, $\vec{f}$ the body forces acting on the fluid, $p$ the pressure and $\sigma$ the stress tensor. However, equation (1) is very complex and, nowadays, we neither know how to solve it analytically, nor whether a solution exists. A numerical approach is possible but heavily computation consuming, which is not to be desired for direct and real-time experiments.

Assuming that the flow is a Newtonian incompressible fluid, hydrodynamic forces are referred as drag force :

$$
\vec{F}_{d}=-\frac{1}{2} \rho\left(u_{r}-v\right)^{2} A C_{d} \frac{\overrightarrow{u_{r}-v}}{\left\|\overrightarrow{u_{r}-v}\right\|}
$$

where $u_{r}-v$ is the relative velocity of the robot with respect to the fluid, $A$ is the frontal area of the core and $C_{d}$ is the drag coefficient.

The drag coefficient represents the overall effect of the robot's shape on the drag force. In the literature, one can find various expressions for the drag coefficient $C_{d}$ as a function of the Reynolds number $R e$. Re is a dimensionless positive number characterizing the flow regime as laminar or turbulent; its expression for a spherical core is given by:

$$
R e=\frac{2 \rho\left(u_{r}-v\right) r}{\eta}
$$

where $\eta$ is the fluid's viscosity.
In our study case, we assume the flow to be pulsatile and laminar, i.e. we neglect the turbulences generated by heart pumping just downstream from the aortic valve. This assumption is not too restrictive if we consider a blood vessel quite far from the heart. In a low Reynolds number, the drag coefficient is approximated by $C_{d}=\frac{24}{R e}$. For higher Reynolds numbers, a more precise drag coefficient in laminar flow for a spherical core is given by [15]:

$$
C_{d}=\frac{24}{R e}+\frac{6}{1+\sqrt{R e}}+0.4
$$

In addition to the drag force, apparent weight (combined action of weight and buoyancy) is acting on the microrobot:

$$
\vec{W}_{a}=V\left(\rho_{r}-\rho\right) \vec{g}
$$

where $V$ and $\vec{g}$ respectively denote the volume of the robot and the gravitational acceleration.

2) Magnetic Force: On the one hand, the permanent magnet of a clinical MRI device commonly generates a strong and uniform magnetic field $\left(B_{0} \geq 1.5 T\right)$, so that we can assume the robot's magnetization reaches the saturation magnetization. This magnetic field induces a torque that tends to align the magnetization of the robot along the field. However, since we consider a spherical bead, it is useless to take it into account.

On the other hand, the gradient coils of the MRI system provide magnetic gradients which produce a magnetic force on the robot. This magnetic force can be expressed as:

$$
\vec{F}_{m}=\mu_{0} V_{m}(\vec{M} . \nabla) \vec{H}
$$

where $\mu_{0}=4 \pi \cdot 10^{-7}$ T.m.A ${ }^{-1}$ is the permeability of free space, $V_{m}$ is the volume of the magnetic material in the core, $\vec{M}$ denotes the magnetization of the core and $\vec{H}$ the external magnetic field, $\nabla$ being the gradient operator.

3) Forces balance: The blood vessel is considered as a tube which divides in two with an angle $\theta$ (Figure 2).

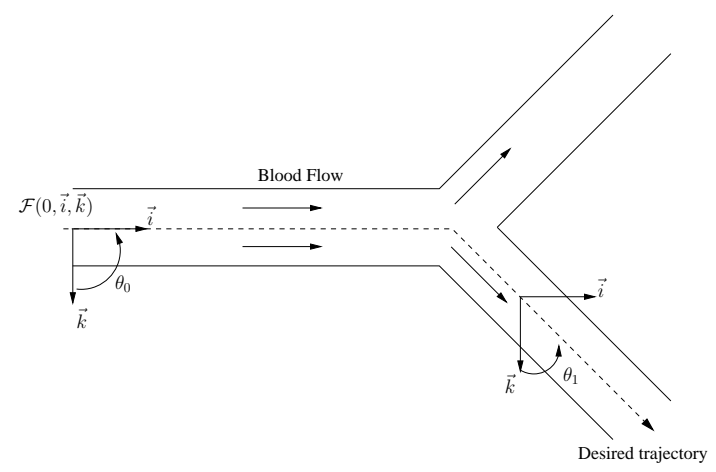

Fig. 2. Scheme of a blood vessel with bifurcation

Let $(x, z)$ denote the location of the robot in the blood vessel with respect to a given frame $\mathcal{F}(0, \vec{i}, \vec{k})$. If we consider that the drag force is distributed linearly between the $\vec{i}$-axis and the $\vec{k}$-axis, applying Newton's second law leads to:

$$
\left\{\begin{array}{l}
m \ddot{x}=\vec{F}_{d_{x}}+\vec{F}_{m_{x}} \\
m \ddot{z}=\vec{F}_{d_{z}}+\vec{W}_{a}+\vec{F}_{m_{z}}
\end{array}\right.
$$


where $m=\frac{4}{3} \pi r^{3} \rho_{r}$ is the mass of the robot, and indexes $x$ (respectively $z$ ) denote the projections on $\vec{i}(\vec{k})$.

If we substitute (2) with (3), (4) and (5) in (6), we get:

$$
\left\{\begin{aligned}
\ddot{x}= & a_{1}\left(\dot{x}-v_{x}\right)+a_{2}\left(\dot{x}-v_{x}\right)^{2} \\
& +a_{3} \frac{\left(\dot{x}-v_{x}\right)^{2}}{1+\alpha \sqrt{\dot{x}-v_{x}}}+a_{4} u_{1} \\
\ddot{z}= & b_{1}\left(\dot{z}-v_{z}\right)+b_{2}\left(\dot{z}-v_{z}\right)^{2}+g\left(\frac{\rho}{\rho_{r}}-1\right) \\
& +b_{3} \frac{\left(\dot{z}-v_{z}\right)^{2}}{1+\alpha \sqrt{\dot{z}-v_{z}}}+b_{4} u_{2}
\end{aligned}\right.
$$

with the following parameters $\alpha, a_{i}, b_{i}$, and magnetic gradients considered as control inputs $u_{1}$ and $u_{2}$ :

$$
\left\{\begin{array}{lll}
a_{1}=-4.5 \frac{\eta \sin \theta}{r^{2} \rho_{r}} & a_{2}=-0.15 \frac{\rho \sin \theta}{r \rho_{r}} & u_{1}=\left\|\vec{\nabla} B_{x}\right\| \\
a_{3}=-2.25 \frac{\rho \sin \theta}{r \rho_{r}} & a_{4}=\frac{M}{\rho_{r}} & u_{2}=\left\|\vec{\nabla} B_{z}\right\| \\
b_{1}=-4.5 \frac{\eta \cos \theta}{r^{2} \rho_{r}} & b_{2}=-0.15 \frac{\rho \cos \theta}{r \rho_{r}} & \alpha=\sqrt{\frac{2 \rho r}{\eta}} \\
b_{3}=-2.25 \frac{\cos \theta}{r \rho_{r}} & b_{4}=\frac{M}{\rho_{r}} &
\end{array}\right.
$$

\section{B. State Space Representation}

Let $x_{1}, x_{2}, x_{3}, x_{4}$ denote respectively the robot's position and velocity along $\vec{i}$-axis, and the position and velocity along $\vec{k}$-axis. Assuming that positions $x_{1}$ and $x_{3}$ can be measured thanks to the MRI imaging system, let $y$ denote the state's measure. Then, system (7) can be written as:

$$
(\mathcal{S})\left\{\begin{array}{ccc}
\dot{x}_{1}= & x_{2} \\
\dot{x}_{2}= & f_{2}\left(x_{2}\right)+\overline{u_{1}} \\
\dot{x}_{3}= & x_{4} \\
\dot{x}_{4}= & f_{4}\left(x_{4}\right)+\overline{u_{2}}
\end{array}\right\}\left(\mathcal{S}_{1}\right)
$$

where functions $f$ and controls $\bar{u}$ are obtained using:

$$
\left\{\begin{array}{ccc}
\overline{u_{1}} & = & a_{2} v_{x}^{2}-a_{1} v_{x}+a_{4} u_{1} \\
\overline{u_{2}} & = & b_{2} v_{z}^{2}-b_{1} v_{z}+g\left(\frac{\rho}{\rho_{r}}-1\right)+b_{4} u_{2} \\
f_{2}\left(x_{2}\right) & = & a_{1} x_{2}+a_{2}\left(x_{2}^{2}-2 v_{x} x_{2}\right)+a_{3} \frac{\left(x_{2}-v_{x}\right)^{2}}{1+\alpha \sqrt{x_{2}-v_{x}}} \\
f_{4}\left(x_{4}\right) & = & b_{1} x_{4}+b_{2}\left(x_{4}^{2}-2 v_{z} x_{4}\right)+b_{3} \frac{\left(x_{4}-v_{z}\right)^{2}}{1+\alpha \sqrt{x_{4}-v_{z}}}
\end{array}\right.
$$

We can notice that system $(\mathcal{S})$ can be divided into two subsystems $\left(\mathcal{S}_{1}\right)$ and $\left(\mathcal{S}_{2}\right)$, what allows to define two independent control laws Lyapunov-stabilizing trajectories for each subsystem.

\section{CONTROL APPROACH}

Each of the two subsystems $\left(\mathcal{S}_{1}\right)$ and $\left(\mathcal{S}_{2}\right)$ can be straight expressed in a triangular form, so that backstepping control approach easily applies [16], [17]. Since the two subsystems expressions are similar, we can focus on the first one. The control law synthesis for the first subsystem can thus be applied mutatis mutandis to the second one.

First, we set $\tilde{x}_{1}=x_{r}-x_{1}$ as the error between any $\mathcal{C}^{2}$ reference trajectory $x_{r}$ and the real position $x_{1}$ of the core. A Lyapunov Control Function (CLF) is given by:

$$
V_{1}\left(\tilde{x}_{1}\right)=\frac{1}{2} \tilde{x}_{1}^{2} \quad \geq 0
$$

Differentiating (9) leads to:

$$
\dot{V}_{1}\left(\tilde{x}_{1}\right)=\frac{\partial V_{1}\left(\tilde{x}_{1}\right)}{\partial \tilde{x}_{1}} \dot{\tilde{x}}_{1}=\tilde{x}_{1}\left(\dot{x}_{r}-x_{2}\right)
$$

To ensure $\dot{V}_{1}\left(\tilde{x}_{1}\right)$ is definite negative, for instance:

$$
\dot{V}_{1}\left(\tilde{x}_{1}\right)=-k_{1} \tilde{x}_{1}^{2}, \quad k_{1} \geq 0
$$

a possible issue is hence to set $-k_{1} \tilde{x}_{1}=\dot{x}_{r}-x_{2 r}$.

Second, setting $\tilde{x}_{2}=x_{2 r}-x_{2}$ implies $\dot{\tilde{x}}_{2}=\ddot{x}_{r}+k_{1} \dot{\tilde{x}}_{1}-\dot{x}_{2}$. Yet we have $\dot{\tilde{x}}_{1}=\dot{x}_{r}-x_{2}=-k_{1} \tilde{x}_{1}$ and $\tilde{x}_{2}=\left(\dot{x}_{r}-x_{2}\right)+$ $k_{1} \tilde{x}_{1}$, hence it follows that $\dot{\tilde{x}}_{1}=\tilde{x}_{2}-k_{1} \tilde{x}_{1}$, and we get:

$$
\dot{\tilde{x}}_{2}=\ddot{x}_{r}+k_{1} \tilde{x}_{2}-k_{1}^{2} \tilde{x}_{1}-\dot{x}_{2}
$$

Using the CLF (9), a Lyapunov function is given by $V_{2}\left(\tilde{x}_{1}, \tilde{x}_{2}\right)=V_{1}\left(\tilde{x}_{1}\right)+\frac{1}{2} \tilde{x}_{2}^{2} \geq 0$. We obtain:

$$
\begin{aligned}
\dot{V}_{2}\left(\tilde{x}_{1}, \tilde{x}_{2}\right) & =\tilde{x}_{1} \dot{\tilde{x}}_{1}+\tilde{x}_{2} \dot{\tilde{x}}_{2} \\
& =-k_{1} \tilde{x}_{1}^{2}+\tilde{x}_{2}\left[\left(1-k_{1}^{2}\right) \tilde{x}_{1}+k_{1} \tilde{x}_{2}+\ddot{x}_{r}-\dot{x}_{2}\right]
\end{aligned}
$$

Likewise, to ensure $\dot{V}_{2}\left(\tilde{x}_{1}, \tilde{x}_{2}\right)$ is definite negative, for instance $\dot{V}_{2}\left(\tilde{x}_{1}, \tilde{x}_{2}\right)=-k_{1} \tilde{x}_{1}^{2}-k_{2} \tilde{x}_{2}^{2}$ with $k_{2} \geq 0$, a possible issue is hence to set:

$$
-k_{2} \tilde{x}_{2}=\left(1-k_{1}^{2}\right) \tilde{x}_{1}+k_{1} \tilde{x}_{2}+\ddot{x}_{r}-\dot{x}_{2}
$$

Substituting expressions of $\tilde{x}_{1}$ and $\tilde{x}_{2}$, and using (8) in the previous expression finally suggests assigning the following control law for subsystem $\left(\mathcal{S}_{1}\right)$ of $(8)$ :

$$
\begin{aligned}
\bar{u}_{1} & =\ddot{x}_{r}+\left(k_{1}+k_{2}\right) \dot{x}_{r}+\left(1+k_{1} k_{2}\right) x_{r} \\
& -\left(1+k_{1} k_{2}\right) x_{1}-\left(k_{1}+k_{2}\right) x_{2}-f_{2}\left(x_{2}\right)
\end{aligned}
$$

\section{HIGH GAIN OBSERVER}

For many applications, appropriate control requires the knowledge of unmeasured parameters, hence the interest in an observer to estimate the state. In our case, we can measure the position of the core but we have also to estimate its velocity, since the control law (10) requires it.

To study the observability of nonlinear system $\dot{x}=f(x)+g(x) u, y=h(x)$, with $x \in \mathbb{R}^{n}, u \in \mathbb{R}^{m}$ and $y \in \mathbb{R}^{p}$, a necessary and sufficient condition is to have a full ranked observability matrix $\mathcal{O}_{i, j}=\frac{\partial L_{f}^{i-1} h(x)}{\partial x_{j}}$, where $L_{f} h(x)$ denotes the Lie derivative of $h(x)$ along the vector field $f(x)$. In system (8), it is clear that the observability is ensured for all $x$.

In our study case, we decided to use a high gain observer. This one requires the system to have canonical form, that is for a second order system:

$$
\left\{\begin{array}{l}
\dot{x}_{1}=x_{2}+\varphi_{1}\left(u, x_{1}\right) \\
\dot{x}_{2}=\varphi_{2}\left(u, x_{1}, x_{2}\right) \\
y=x_{1}
\end{array}\right.
$$

According to [18], if $\mid \varphi_{k}\left(u, x_{1}, \ldots, x_{k}\right)-$ $\varphi_{k}\left(u, \hat{x}_{1}, \ldots, \hat{x}_{k}\right) \mid \leq c \sqrt{\left(x_{1}-\hat{x}_{1}\right)^{2}+\ldots+\left(x_{k}-\hat{x}_{k}\right)^{2}}$
where $c$ is a locally Lipschitz constant, then an observer is given by:

$\left\{\begin{array}{l}\dot{\hat{x}}_{1}=\hat{x}_{2}+\varphi_{1}\left(u, \hat{x}_{1}\right)+L g_{1}\left(\hat{x}_{1}-x_{1}\right) \\ \dot{\hat{x}}_{2}=\varphi_{2}\left(u, \hat{x}_{1}, \hat{x}_{2}\right)+L^{2} g_{2}\left(\hat{x}_{1}-x_{1}\right)\end{array} \quad, H=\left(\begin{array}{ll}g_{1} & 1 \\ g_{2} & 0\end{array}\right)\right.$

where $L$ is the high gain and with $H$ a Hurwitz matrix. 


\section{SIMULATIONS}

Simulations are conducted within the scope of actual commonly spread MRI system abilities. At the moment, MRI systems are able to generate magnetic gradients with an intensity of some tens of $m T \cdot m^{-1}$. Let us note that this limitation is additionally affected by the gradient coils' duty cycle and by the multiplexing needed both for controlling and observing. That is the reason why some prospects are actually led in order to design additional powerful gradient coils [19], which is a difficult task since they must not affect the imaging capability and quality. In order to make sure that the amplitude of the control inputs remains bounded by physical actuators limits $u_{i, \max }$ and to protect the system, we perform a simple time scaling. Thus, the control is first computed using (10), and the applied control law is given by $\frac{u_{i}}{k(t)}$, with $k(t)=\max \left\{1, \frac{u_{i}}{u_{i, \max }}\right\}$.

TABLE I

Simulations Data

\begin{tabular}{|c||c||c|}
\hline Radius of the core & $r$ & $300 \mu \mathrm{m}$ \\
\hline Blood's viscosity & $\eta$ & $15 \times 10^{-3} \mathrm{Pa.s}$ \\
\hline Blood's density & $\rho$ & $1060 \mathrm{~kg} \cdot \mathrm{m}^{-3}$ \\
\hline Bead's density & $\rho_{\text {sphere }}$ & $8000 \mathrm{~kg} . \mathrm{m}^{-3}$ \\
\hline Magnetization & $M$ & $1.950 \times 10^{6} \mathrm{~A} . \mathrm{m}^{-1}$ \\
\hline Angle & $\theta$ & $\frac{\pi}{2}$ \\
\hline Initial condition on $x$ & $x_{0}$ & $(0,0,0,0)^{T}$ \\
\hline Initial condition on $\hat{x}$ & $\hat{x}_{0}$ & $(0.01,-0.01,0.01,-0.01)^{T}$ \\
\hline Inputs saturations & $u_{i, \max }$ & $45 \mathrm{mT} . \mathrm{m}^{-1}$ \\
\hline Controller gains & $\left(k_{1}, k_{2}\right)$ & $(50,100)$ \\
\hline Observer gains & $\left(g_{1}, g_{2}, L\right)$ & $(-5,-5,400)$ \\
\hline
\end{tabular}

We suppose that a pre-planned trajectory has been obtained, and the control aims at keeping a null error between desired and real trajectory. The following simulations illustrate two different backgrounds. In the first one, we consider that the reference trajectory requires the robot to move with and against the flow and study effects of noise measurements on tracking. In the latter, a 2D Y-shaped reference trajectory is considered to validate our approach, with uncertainties considerations. Simulation parameters are given in Table I.

\section{A. ID trajectory with and against the flow}

This simulation is performed only on the $\vec{i}$-axis, which does not affect the 2D-approach we lead since the two subsystems are similar and so for the results. The only difference is that, on $\vec{k}$-axis, the gravity must be counterbalanced in order to levitate the ferromagnetic core.

Reference trajectory is here a sinusoidal function $x_{r}(t)=$ $0.01 \sin \frac{5.5}{2} t$ along the $\vec{i}$-axis, thus illustrating the case of swimming longitudinally with and against the flow. The noise applied on the output is a white gaussian noise with a $10^{-4}$ amplitude, i.e. about $10 \%$ of the measured signal. The blood's velocity $v=0.035(1+1.15 \sin 2 \pi t)$ is supposed to be known. Since blood's velocity is periodic, using the previous expression is relevant for simulation's purpose, even if not realistic.

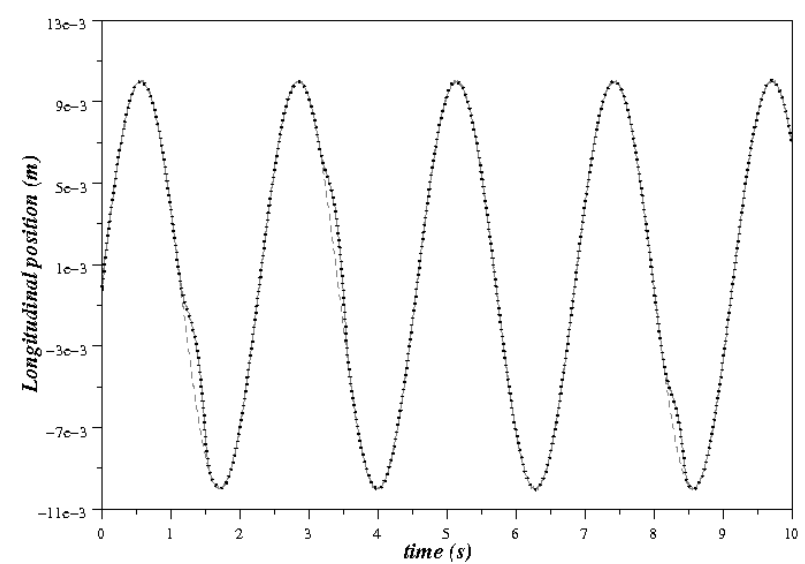

Fig. 3. Longitudinal position: reference position (grey dots), actual position (black dots) and estimated position (grey solid line)

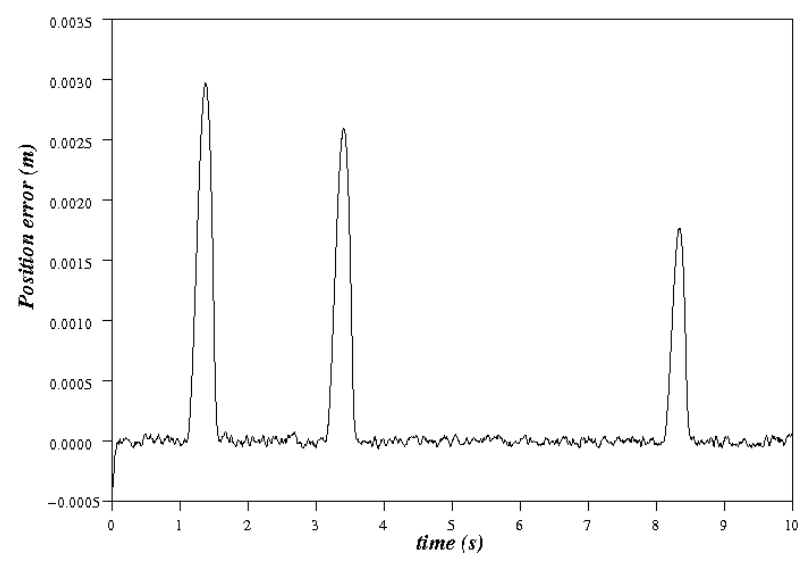

Fig. 4. Error between actual and reference position

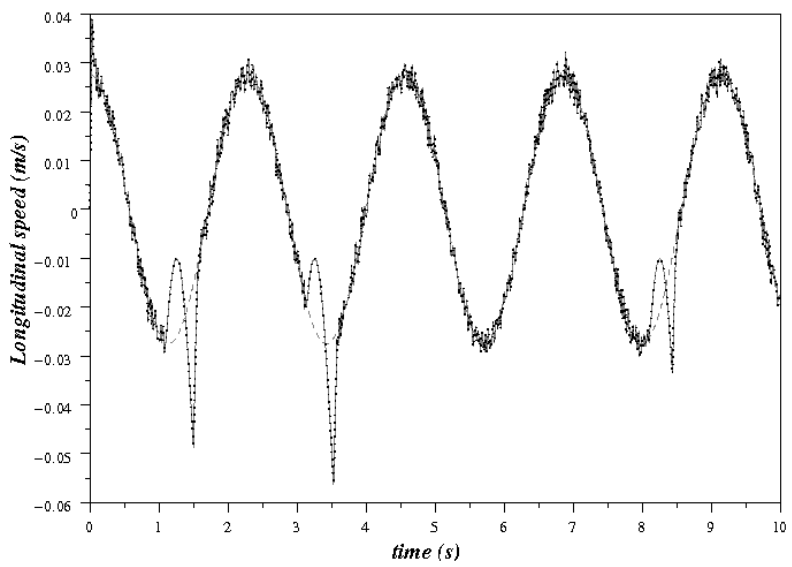

Fig. 5. Longitudinal speed: reference speed (grey dots), actual speed (black dots) and estimated speed (grey solid line)

Figures 3 and 4 illustrate that the position tracking is good with respect to noise and the observer's convergence is efficient. Globally, tracking is not too much affected by the noise, since position error is below $0.1 \%$ of the reference position, and the observer-controller pair ensures an efficient stabilization after a short transient phase. Note that notable degradation of tracking performance occurs at times $t \in$ $\{1 ; 3 ; 8\}$, because the control input reaches low saturation at these times (Figure 7).

Figures 5 and 6 also show a rather good tracking of 


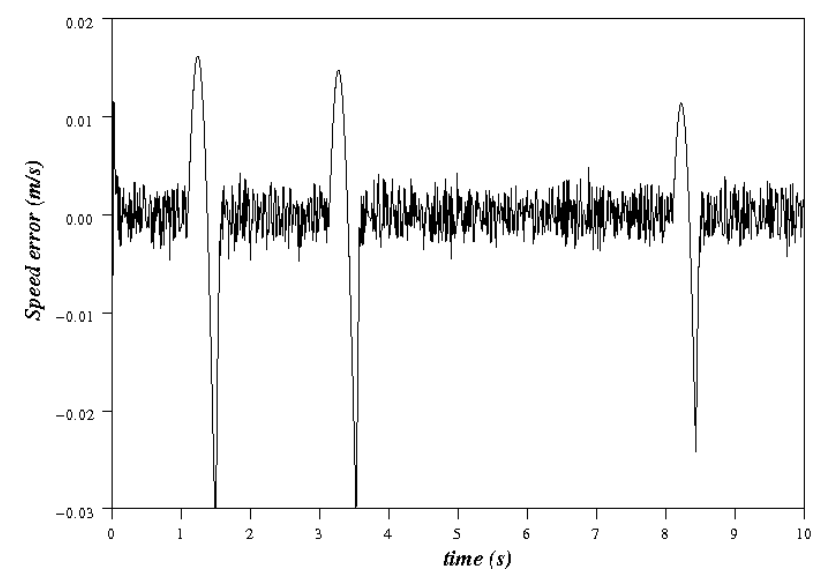

Fig. 6. Error between actual and reference speed

velocity, yet not as efficient as the position tracking, since the noise induced on speed error is about $2 \%$ of desired speed. This result should be expected, as the estimate velocity highly depends on the noised measures on position. We can also notice the three peaks on speed error related with control's saturation.

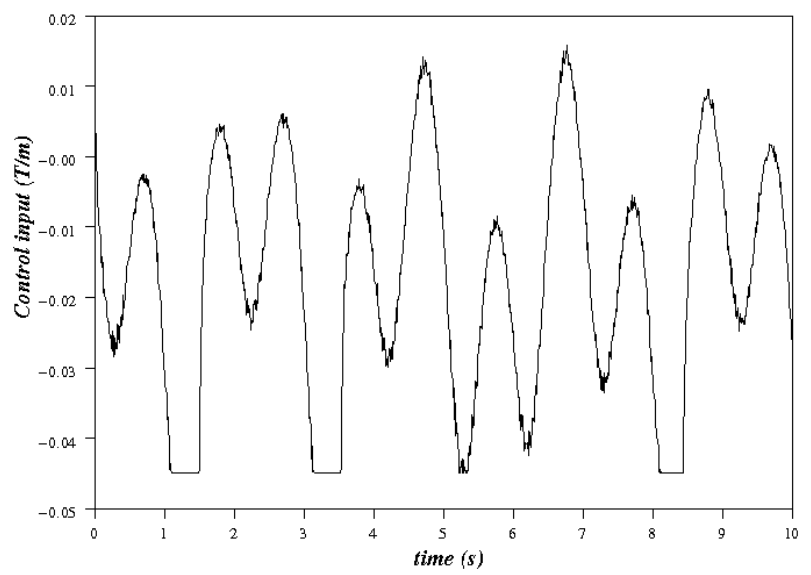

Fig. 7. Control input (magnetic field gradient on $\vec{i}$-axis)

\section{B. 2D Y-shaped trajectory}

In order to illustrate any case of Y-shaped bifurcation, simulation is led so as to work in every quadrant using a circular reference trajectory (Figure 8). Experiment in the 2D space defined by the frame $\mathcal{F}(0, \vec{i}, \vec{k})$ of Figure 2 is of particular interest, since the special issue of levitating along $\vec{k}$-axis is encompassed as well as less demanding control on $\vec{i}$ (or $\vec{j}$ ) axis.

Moreover, the blood's velocity $v=0.035(1+1.15 \sin 2 \pi t)$ is unknown at the controller-observer level. This matched additive uncertainty on the physical system is motivated by the difficulty to measure the pumping blood stream in the vessels, whereas it is a decisive parameter in the force balance. We consider here the worst case scenario, since this parameter is considered as null when designing the control as well as the observer.

Figures 8 and 9 show that the position tracking performance is quite robust to unmodeled dynamics. After convergence of both controller and observer, tracking error is fewer

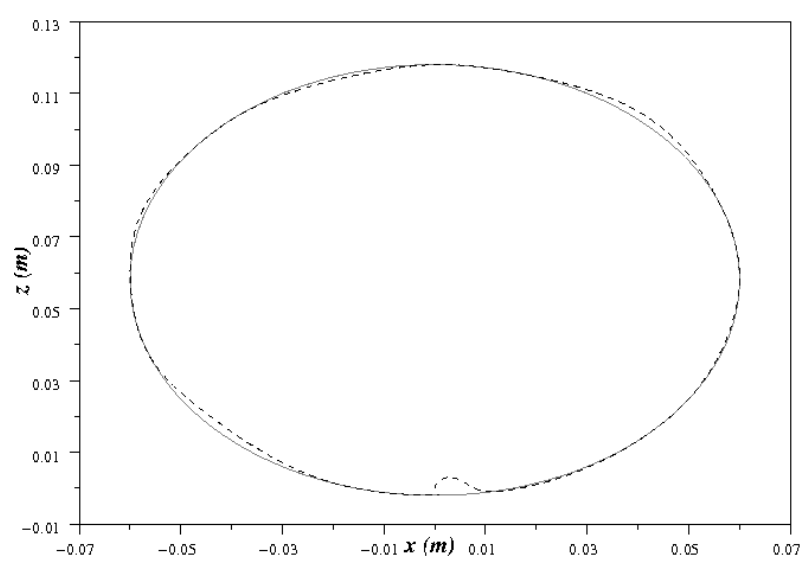

Fig. 8. XY trajectory: reference trajectory (grey) and actual trajectory (black dotted)

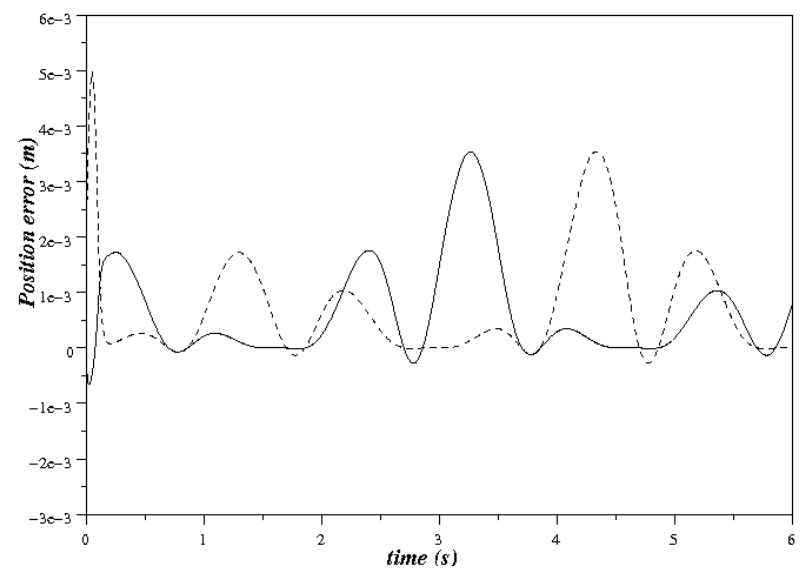

Fig. 9. Position error between actual and desired position: on $\vec{i}$-axis (solid) on $\vec{k}$-axis (dotted)

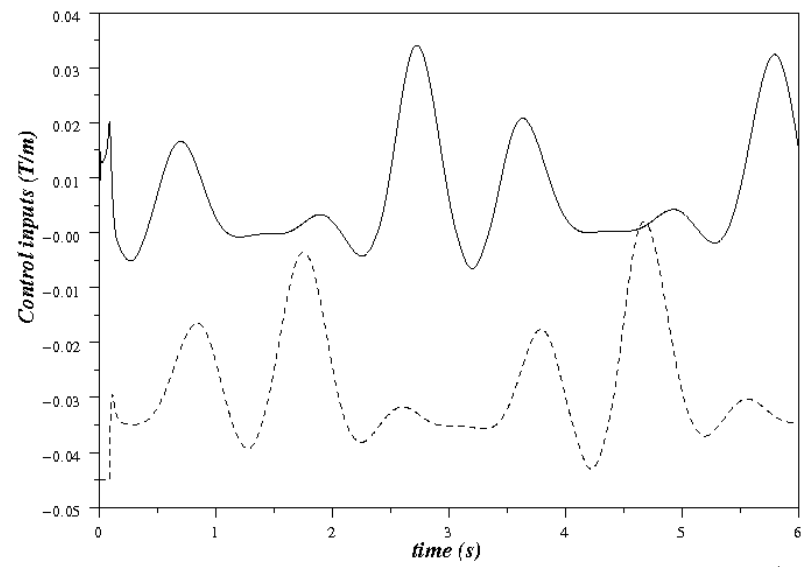

Fig. 10. Control inputs: magnetic field gradients on $\vec{i}$-axis (solid), on $\vec{k}$-axis (dotted)

than $4 \mathrm{~mm}$ for each of the considered axis, suggesting that bifurcating can be performed even with important fluctuating uncertainties, at least for vessels whose diameter is up to some $5 \mathrm{~mm}$. However, to navigate efficiently in smaller vessels, this result suggests it would be necessary either to reject this unknown perturbation, or to rebuilt it.

Figure 10 shows the gradient inputs on $\vec{i}$ and $\vec{k}$-axis. During transient phase, control input on $\vec{k}$-axis reaches its actuator saturation, which explains the peaks at the beginning of the simulation. It can be noticed that input on the vertical 
magnetic gradient is mainly negative: this is due to the imposed constraint of levitating, which is directly related to a force balance between apparent weight versus magnetic force. For the parameters values given in Table I, levitating requires about $\nabla B_{\text {lev }}=-\frac{\left(\rho_{\text {sphere }}-\rho\right) g}{M} \approx-36 m \mathrm{Tm}^{-1}$, which is at the merge of control saturation.

\section{DISCUSSION}

The previous simulations show that it is possible to control a some hundred micrometers radius ferromagnetic core in a blood vessel in 1D and 2D (with no particular difficulty to extend it to 3D), with an improved efficiency compared to PID controllers. In the first case, we show that our controller-observer is robust to output noise. In fact, actual MRI devices have a precision around some hundreds of micrometers, besides the microrobot being ferromagnetic, it may induce artifacts on the MRI imager. That is why output noise robustness is a key point for implementation on such process.

The second simulation points out the relative robustness of the proposed controller-observer with respect to some unmodeled dynamics. Since microrobots are mainly devoted to medical applications, controllers designed for such robots have to deal with well-known uncertain biological systems.

\section{CONCLUSION AND FUTURE WORK}

In this paper, we have presented a state space representation of a magnetic microrobot in a fluidic environment and have developed both a nonlinear control law and an observer to improve actual experiments. We have studied the effect of noise on the output and its influence on the position and speed tracking as well as the convergence of the high gain observer. We have remarked that the position is not too much affected by the noise, unlike the velocity of the core. We also have showed that the proposed design is robust with respect to some matched uncertainties. However, we noticed that tracking efficiency is very sensitive to some biological parameters uncertainties. Besides, robustness to blood's velocity unknowing reaches its limits in the case of small vessels.

In near future, we can expect better performances by improving the controller, using adaptative rejection of matched uncertainties based on [20]. Another promising improvement may be obtained using the varying high gain observer proposed in [21], which allows, under some conditions, to take into account these perturbations and to ensure the practical convergence of the observer.

Beyond theoretic and simulating developments we are working on, we expect to soon implement these nonlinear tools on the benchmark MR-SUB of the Nanorobotics Laboratory through afoot collaboration with the Ecole Polytechnique of Montreal.

\section{ACKNOWLEDGMENTS}

This work was supported by European Union's 7th Framework Program and its research area ICT-2007.3.6 Micro/nanosystems under the project NANOMA (NanoActuactors and Nano-Sensors for Medical Applications.)

\section{REFERENCES}

[1] J. Abbott, Z. Nagy, F. Beyeler, and B. Nelson, "Robotics in the small: Part 1 microrobotics," IEEE Robotics and Automation Magazine, vol. 14, no. 2, pp. 92-103, June 2007.

[2] G. T. Gillies, R. C. Ritter, W. C. Broaddus, M. S. Grady, M. A. Howard, and R. G. McNeil, "Magnetic manipulation instrumentation for medical physics research," Rev. of Sci. Instruments, vol. 65, no. 3, March 1994.

[3] T. Honda, K. I. Arai, and K. Ishiyama, "Micro swimming mechanisms propelled by external magnetic fields," IEEE Trans. on Magnetics, vol. 32, no. 5, 1996.

[4] E. G. Quate, K. G. Wika, M. A. Lawson, G. T. Gillies, R. C. Ritter, M. S. Grady, and M. A. H. and, "Goniometric motion controller for the superconducting coil in amagnetic stereoaxis system," IEEE Trans. on Biomed. Eng., vol. 38, no. 9, pp. 899-905, September 1991.

[5] S.-I. Takeda, F. Mishima, S. Fujimoto, Y. Izumi, and S. Nishijima, "Development of magnetically targeted drug delivery system using superconducting magnet," Journal of Magnetism and Magnetic Materials, 2006.

[6] J.-B. Mathieu, G. Beaudoin, and S. Martel, "Method of propulsion of a ferromagnetic core in the cardiovascular system through magnetic gradients generated by an mri system," IEEE Trans. on Biomed. Eng., vol. 53, no. 2, February 2006.

[7] J.-B. Mathieu and S. Martel, "Magnetic microparticle steering within the constraints of an mri system: proof of concept of a novel targeting approach," Biomedical Microdevices, vol. 9, no. 6, December 2007.

[8] S. Tamaz, R. Gourdeau, A. Chanu, J.-B. Mathieu, and S. Martel, "Real-time mri-based control of a ferromagnetic core for endovascular navigation," IEEE Trans. on Biomed. Eng., vol. 55, no. 7, July 2008.

[9] S. Martel, J.-B. Mathieu, O. Felfoul, A. Chanu, E. Aboussouan, S. Tamaz, P. Pouponneau, H. Yahia, G. Beaudoin, G. Soulez, and M. Mankiewicz, "Automatic navigation of an untethered device in the artery of a living animal using a conventional clinical magnetic resonance imaging system," Applied Physics Letters, vol. 90, no. 11, 2007.

[10] R. Dreyfus, J. Beaudry, M. L. Roper, M. Fermigier, H. A. Stone, and J. Bibette, "Microscopic artificial swimmers," Nature, vol. 437, pp. 862-865, October 2005.

[11] B. Behkam and M. Sitti, "Design methodology for biomimetic propulsion of miniature swimming robots," ASME J. of Dynamic Systems, Measurement, and Control, vol. 128, no. 1, 2006.

[12] J. J. Abbott, K. E. Peyer, M. C. Lagomarsino, L. Zhang, L. Dong, I. K. Kaliakatsos, and B. J. Nelson, "How should microrobots swim?" in Int. Symp. of Robotics Research, November 2007.

[13] S. Martel, M. Mohammadi, O. Felfoul, Z. Lu, and P. Pouponneau, "Flagellated magnetotactic bacteria as controlled mri-trackable propulsion and steering systems for medical nanorobots operating in the human microvasculature," IJRR, to be published.

[14] J. Mathieu and S. Martel, "In-vivo validation of a propulsion method for untethered medical microrobots using a clinical magnetic resonance imaging system," in IROS, 2007, pp. 502-508.

[15] F. White, Viscous Fluid Flow. McGraw Hill New-York, 1991.

[16] I. Kanellakopoulos, P. Kokotović, and A. Morse, "A toolkit for nonlinear feedback design,” $S C L$, vol. 18, pp. 83-92, 1992.

[17] M. Krstić, I. Kanellakopoulos, and P. Kokotović, Nonlinear and Adaptative Control Design. John Wiley \& Sins, Inc., New York, 1995.

[18] J. Gauthier, H. Hammouri, and S. Othman, "A simple observer for nonlinear systems. application to bioreactors." ITAC, vol. 37, no. 6 , pp. 875-880, 1992.

[19] K. B. Yelin, K. Vollmers, and B. J. Nelson, "Modeling and control of untethered biomicrorobots in a fuidic environment using electromagnetic fields," IJRR, vol. 25, no. 5-6, pp. 527-536, May-June 2006.

[20] A. Chakrabortty and M. Arcak, "Time-scale separation redesigns for stabilization and performance recovery of uncertain nonlinear systems," Automatica, vol. 45, no. 1, pp. 34-44, 2009.

[21] V. Andrieu, L. Praly, and A. Astolfi, "High gain observers with updated gain and homogeneous correction terms," Automatica, vol. 45, no. 2, pp. 422-428, January 2009 . 